\title{
Astronomy Education: an international perspective
}

\author{
By John R. Percy \\ Erindale Campus, University of Toronto, Mississauga ON, Canada L5L 1C6
}

As the first speaker at this Colloquium, it is my pleasure to welcome the participants (and the readers of these Proceedings), on behalf of the International Astronomical Union (IAU) and its Commission 46 (Teaching of Astronomy). It is also my pleasure to thank our hosts University College London, and The Open University; the Scientific Organizing Committee, chaired by Lucienne Gouguenheim, and especially the Local Organizing Committee, chaired by Barrie Jones and Derek McNally. They have made this meeting most enjoyable and successful.

Eight years ago, many of us were in Williamstown, USA, for the first IAU Colloquium on astronomy education. Since then, there have been enormous changes - political, economic, and technological - which have affected our work. There have also been about 100 IAU conferences on research topics, but this is only the second on education. We all agree that we must work to correct that imbalance!

We are here to catch up on what has happened in astronomy education in the last eight years. We are here to teach and learn, through lectures, posters, and discussions - both formal and informal. We are here to renew old friendships, and make new ones. These human dimensions of this Colloquium are only hinted at in these Proceedings, but I assure you that they occurred.

\section{Why is Astronomy Education Important?}

Education is important to astronomers because it affects the recruitment and training of future astronomers, and because it affects the awareness, understanding and appreciation of astronomy by taxpayers and politicians who support us. We have an obligation to share the excitement and the significance of our work with students and the public. Education is often neglected by the scientific and professional community - not by us, of course - and by many research universities. Our task is not only to be better astronomy educators ourselves, but to convince and train our students and colleagues to do likewise.

There are other reasons why astronomy should be part of our education system and our culture. Astronomy is deeply rooted in the history of almost every society, as a result of its practical applications and its philosophical implications. It still has everyday applications to timekeeping, seasons, navigation and climate, as well as to longer-term issues such as climate change and biological evolution. Astronomy not only contributes to the development of physics and the other sciences, but it is an important and exciting science in its own right. It deals with the origin of the stars, planets, and life itself. It shows our place in time and space, and our kinship with other peoples and species on earth. It reveals a universe which is vast, varied and beautiful. It promotes curiosity, imagination, and a sense of shared exploration and discovery. It provides an enjoyable hobby for millions of people, whether they be serious amateur astronomers, armchair astronomers, or casual skygazers. In a school context, it demonstrates an alternative approach to the "scientific method" - the observation vs. theory approach. It can attract young people to study science and engineering, and can increase public interest and understanding of science and technology - both of which are important in all countries, both developed and developing. 


\section{What is Astronomy Education?}

It is important, before we go further, to define astronomy education. I propose to define it broadly, by quoting Andrew Fraknoi (1996), who posed the following question: "Where does astronomy education take place? Those readers who teach will probably say that it takes place in classrooms like theirs - anywhere from first grade through university. But I want to argue that astronomy education happens in many other places besides the formal classroom. It happens in hundreds of planetaria and museums. It happens at meetings of amateur astronomy groups. It happens when someone reads a newspaper, or in front of television and radio sets. It happens when someone is engrossed in a popular book on astronomy, or leafs through a magazine like Sky and Telescope. It happens in youth groups taking an overnight hike, and learning about the stars. It happens when someone surfs the astronomy resources on the Internet. When we consider astronomy education, its triumphs and tribulations, we must be sure that we do not focus too narrowly on academia, and omit the many places that it can and does happen outside the classroom."

I believe that our students are influenced by the astronomy which they learn outside the classroom, as much or more than by the astronomy which they learn from us. Anderson (1996) was recently appalled to find that his college astronomy students, writing essays, did not hesitate to quote popular books and TV programs on pseudoscience as authorities! It is unfortunate that we do not have more educators here from planetariums, science centres, publishers, the news media, and amateur astronomy. Perhaps, at the next IAU Colloquium on education, we can devote much more time to the issue of "informal education".

\section{The Problems of Astronomy Education}

If astronomy is so interesting and important, and available in so many settings, why is it not taught in more schools? Why are there so many misconceptions about astronomical topics? Astronomy educators all over the world have discovered "universal" barriers to the effective teaching and learning of astronomy. Some of them have discovered solutions!

(a) Students have misconceptions about astronomical topics (such as the causes of the seasons), which are not overcome by the teaching methods which are commonly used.

(b) Teachers have misconceptions about the teaching of astronomy (and perhaps about astronomical content as well) which discourage them from teaching it well, or teaching it at all.

(c) Teachers (especially in elementary schools) have very little knowledge about astronomy or astronomy teaching, while astronomy has progressed by leaps and bounds since these teachers were in school themselves.

(d) The most effective teaching tools are simple, inexpensive, hands-on activities, and these are not widely used, or not widely available. These must get around the problem that "the stars come out at night, but the students don't". But what is expensive for the North American teacher is beyond the reach of teachers in the developing world, so we will have to emphasize the use of "available materials" for teaching.

(e) Teachers are not always aware of the materials available. There is not a single national or international journal which deals specifically with astronomy education, or an effective network for informing teachers of materials available. The development of the European Association for Astronomy Education is a step in the right direction. It will make good use of the Internet for communication, but we must remember that the Internet is still not available to the vast majority of teachers around the world. 
(f) The best or most fortunate students may receive good education, but the others girls, minorities, the disabled, inner-city or rural students - and students in the developing countries - may be left out.

(g) The best students must be attracted to astronomy and astronomy education; and provided with career opportunities when they graduate.

(h) Teaching often has less status and support than research. Ironically, the problem in elementary school is often that administrators ignore astronomy. In university, they ignore education!

Note that these barriers apply, to a greater or lesser extent, at all levels of education, and in all countries of the world. For instance, even in graduate education, there is a need for professional development of instructors and supervisors. There is debate about the relative importance of coursework and practical work. There is a need to attract women and minorities, and a real need to prepare students for jobs in astronomy and elsewhere.

We need to take a wider look at astronomy education, and its problems. There are innovative projects and programs in many countries, in many cases well-tested. By taking an international perspective, we achieve a deeper historical and cultural understanding, which is especially important in our multicultural societies. We must also work in partnership with teachers, educators in planetariums and science centres, amateur astronomers, and all others who contribute to astronomy education in its broadest sense. We take some pleasure in knowing that, as astronomy educators, we have "kindred spirits" around the world and, in the case of the developing countries, we take some satisfaction in knowing that we can help scientists and educators less fortunate than ourselves.

\section{Varieties of Astronomy Education}

Even within formal or classroom astronomy education, there are many varieties as you will quickly learn at this meeting. There are the two systems of education eloquently described by Don Wentzel (1990) in the introductory lecture at the last IAU Colloquium on astronomy education - the European system, and the North American system. There are also two (or more) methods of education: at one end of the spectrum is the method based on the memorization of lecture and textbook material; at the other end is the inquiry-based or hands- on approach. There are many opinions about astronomy education curriculum: one is to teach a course or unit on general astronomy; another is to introduce astronomy as part of other sciences such as physics. If astronomy is taught as a course unit, should it emphasize classical content or current developments, core concepts or interdisciplinary or speculative topics; topics relevant to everyday or abstract topics like black holes; selective or comprehensive coverage; depth or breadth? If there is practical work, should it be sky-based, or computer-based? If sky-based, should it be daytime or nighttime? Or should there be elements of all of the above?

\section{The Needs of the Developing Countries}

Whichever systems and methods are used, the developing countries face problems not encountered by astronomy educators elsewhere. Their special needs include: an understanding and interest on the part of astronomy educators elsewhere; opportunities to gain experience in more developed countries, and be visited by astronomers and educators from abroad; access to appropriate books, journals, and data; access to equipment. 
Many of the programs and projects of the International Astronomical Union, described elsewhere in this meeting, address these needs.

I use the term "developing countries" broadly. Of the almost 200 states which are recognized by the United Nations, about 100 have some form of organized astronomical activity - either professional or amateur. About half those countries adhere to the IAU. Of those, less than half could be considered fully developed, (considering that the countries of Eastern Europe and the former Soviet Union still face many economic obstacles, despite their rich astronomical heritage). I am including Canada and the US among the developed countries, even though astronomers there have many concerns about the quality of their education system!

In many developing countries, there is only one "lone astronomer" (at most a small group) to do all the educational, research and administrative work which is shared among many in the more developed countries. The accomplishments of these individuals are remarkable. They should be an inspiration to all of us. I am glad that a few of them are here at this Colloquium.

\section{Astronomy Education Around The World}

It is tempting to try to review all aspects of astronomy education, at all levels, in all countries, but I shall resist the temptation. You will learn all you want to know from this meeting, and from the people who are here. For further reading, I can refer you to "The Teaching of Astronomy" Pasachoff and Percy (1990), the Proceedings of the last IAU Colloquium on education, as well as to the several articles which $I$ have written in the course of my work with IAU Commission 46 (Percy 1994, 1995abc, 1996a).

In the Colloquium, at this point, I showed a large number of slides of astronomy education around the world. They illustrated the education projects and programs of the IAU, as well as local initiatives - some of which are described at this meeting.

\section{How Can Astronomers and Educators Support and Improve Education?}

(a) Make education a part of the professional astronomy organization in your country, and part of your department, observatory or local astronomical society:

- appoint an education co-ordinator, and an education committee

- include an education column in your journal, and education sessions at your annual meeting

- sponsor public lectures and teachers' workshops as part of your annual meeting

- work with other organizations and institutions which have an interest in astronomy education in your area, and in your country.

(b) Be aware of developments in astronomy and science education:

- be aware of curriculum changes in your country, and make sure that astronomy is not neglected

- adopt existing astronomy education materials when possible, instead of "rediscovering the wheel".

- organize meetings on astronomy education, inviting all those who are involved in that subject.

(c) Seek more funds for science education, from governments and private sources.

(d) Make a contribution to astronomy education, and support others who do:

- pass on your knowledge and enthusiasm 
- publicize the practical and cultural benefits of astronomy

- give or arrange a public lecture or other such event

- write a popular article on astronomy

- meet with students, teachers and the public

- encourage interested students, especially women and other under-represented groups

- support your local elementary and secondary school

(e) Get more and better astronomy in:

- museums, planetaria and science centres

- parks and conservation areas

- day schools and night schools

- the news media: newspapers, magazines, radio and TV.

(f) Lobby for, and help develop:

- a planetarium

- a science centre

- a public observatory.

(g) Support astronomy education in the developing countries:

- learn about the needs of these countries

- support programs to send surplus books, journals and instruments to these countries

- communicate with, visit, and support the work of the "lone astronomers" in the developing countries

- support the work of the IAU.

This seems like a daunting list, especially when read all at once. I do not ask astronomers to devote all their time to education (unless, of course, that is what they are paid to do). If each astronomer were to spend at least 10 per cent of their time on education, having made a conscious effort to learn more about astronomy education, and to co-ordinate their work with that of others, the results would be quite remarkable! In a recent special issue of the Astronomical Society of the Pacific's popular magazine Mercury, on the future of astronomy, I published a rather optimistic view of the future of astronomy education (Percy 1996b). I urge you to read that article, and help make my dream come true!

\section{REFERENCES}

ANDERSON, W.R., 1996, Sceptical Inquirer 20, 5, 59-60.

Pasachoff, J.M. And Percy, J.R. (editors) 1990, "The Teaching of Astronomy", Cambridge, Cambridge University Press.

Percy, J.R., 1994, Southern Stars, 35, 223-230.

PERCY, J.R., 1995a, The Planetarian 14, 3, 6-9.

PERCY, J.R., 1995b, MNASSA 54, 38-40.

Percy, J.R., 1995c, Fifth International Conference on Teaching Astronomy ed. R.M. Ros, Universidad Politecnica de Catalunya, 63-68.

PERCY, J.R., 1996, Astronomy Education: Current Developments, Future Co-ordination, ed. J.R. Percy, ASP Conf. Series, Vol. 89, 1-8.

PeRCY, J.R., 1996b, Mercury 25, 1, 34-36. 